\title{
Efeito da Severidade de Oídio e Crestamento Foliar de Cercospora na Produtividade da Cultura da Soja
}

\author{
Cláudia V. Godoy ${ }^{1}$ \& Marcelo G. Canteri $^{2}$ \\ ${ }^{1}$ Embrapa Soja, Cx. Postal 231, CEP 86001-970, Londrina, PR, fax: (43) 3371-6100, e-mail: godoy@cnpso.embrapa.br; \\ ²Universidade Estadual de Londrina, CEP 86051-990, Londrina, PR, e-mail: canteri@uel.br
}

(Aceito para publicação em 08/07/2004)

Autor para correspondência: Cláudia Vieira Godoy

GODOY, C.V. \& CANTERI, M.G. Efeito da severidade de oídio e crestamento foliar de cercospora na produtividade da cultura da soja. Fitopatologia Brasileira 29:526-531. 2004.

\section{RESUMO}

Ensaios de campo foram conduzidos na safra 2002/03, com as cultivares de soja (Glycine max) BRS 133 e BR 16, visando quantificar o efeito das doenças foliares na produtividade da cultura. O delineamento experimental foi blocos ao acaso com seis tratamentos (diferentes níveis de doença) e quatro repetições, sendo cada repetição formada por parcelas de $18 \mathrm{~m}^{2}$. Diferentes épocas e números de aplicações de fungicida (difenoconazole + propiconazole) foram utilizados para criar um gradiente de doença. Foram realizadas avaliações semanais da severidade de doenças e do índice de área foliar, utilizando o analisador de dossel $\left(\mathrm{Li}-\mathrm{Cor}^{\circledR}\right)$. A produtividade foi quantificada nos $4 \mathrm{~m}^{2}$ centrais das parcelas. As doenças predominantes no ensaio foram oídio e crestamento de cercospora. A severidade de doença, nos estádios R6 (vagens com sementes verdes que preenchem totalmente a cavidade), e R7 (início a 50\% de amarelecimento de folhas e vagens) apresentou uma correlação negativa significativa com a produtividade, utilizando-se regressão linear. As variáveis integrais área abaixo da curva de progresso da doença (AACPD), obtida a partir da integração das curvas de progresso das doenças, e duração da área foliar sadia (DAFS), obtida a partir da integração do índice de área foliar sadia, foram relacionadas à produtividade, havendo correlação negativa com AACPD, utilizandose regressão linear $\left(\mathrm{R}^{2}=0,67\right.$ para BRS 133 e 0,69 para BR16) e positiva com DAFS, utilizando-se o modelo monomolecular $\left(\mathrm{R}^{2}=0,76\right.$ para BRS 133 e 0,62 para BR 16).

Palavras-chave adicionais: duração da área foliar sadia, Erysiphe diffusa, Cercospora kikuchii.

\section{ABSTRACT}

Effect of powdery mildew and cercospora blight severity on soybean yield

Field trials were carried out in 2002/03, with soybean (Glycine max) cultivars BRS 133 and BR 16, to evaluate the effect of foliar diseases on yield. The experimental design was complete randomized blocks with six treatments (different levels of disease) and four replications with plots of $18 \mathrm{~m}^{2}$. Different times and numbers of applications of fungicide (difenoconazole + propiconazole) were used to generate a disease gradient. Disease severity and leaf area index were assessed weekly. Yield was estimated on central rows of the

\section{INTRODUÇÃO}

A soja [Glycine max (L.) Merrill] é considerada a mais importante fonte de proteína e óleo vegetal no mundo, em função da qualidade e do baixo custo de produção. O desenvolvimento de novas cultivares adaptadas aos trópicos e a geração de novas tecnologias contribuiu para que o Brasil aumentasse sua produção, com valor estimado na safra 2002/ 03, de 52,2 milhões de ton de grãos em 18.534 milhões ha. Apesar do grande esforço e dos recursos investidos na pesquisa e na assistência técnica, a produtividade brasileira da soja, na safra 2002/03, foi de 2.817 kg/ha (CONAB, 2003), quando o potencial, na prática, é de $3.600 \mathrm{~kg} / \mathrm{ha}$. Entre os fatores que contribuem para a variação no rendimento da cultura nas diversas regiões estão as doenças. Aproximadamente 40 plots. Powdery mildew and cercospora blight were the predominant diseases in these trials. Disease severity at R6 (pods with full sized green beans) and R7 (pods yellowing, 50\% of leaves yellowing) presented a significant negative linear correlation with yield. The relationship between the area under the disease progress curve (AUDPC) and healthy leaf area duration (HAD) and yields were investigated. The AUDPC presented a negative linear correlation with yield $\left(\mathrm{R}^{2}=0.67\right.$ for BRS 133 and 0.69 for BR16) and a positive correlation with HAD, when a monomolecular model was used $\left(\mathrm{R}^{2}=0.76\right.$ for BRS 133 and 0.62 for BR 16).

doenças causadas por fungos, bactérias, nematóides e vírus já foram identificadas no Brasil. A importância econômica de cada doença varia de ano para ano e de região para região, dependendo das condições climáticas de cada safra (Embrapa, 2002).

A estimativa de danos causados por doenças, na cultura da soja, tem sido obtida pela simples diferença entre a produção de parcelas sadias e produção de parcelas ou plantas doentes. Métodos tradicionais de determinações de danos (método do ponto crítico, múltiplos pontos e superfície de resposta), onde a intensidade da doença é correlacionada a componentes de produção (James \& Teng, 1979), usuais em outras culturas (James et al, 1968), têm sido pouco utilizados na cultura da soja. Dessa forma, os mais diversos índices de redução de produtividade são relatados para diferentes doenças. Danos 
Efeito da severidade de oídio e crestamento foliar de cercospora ...

devidos à mancha alvo [Corynespora cassiicola (Berk. \& Curt) Wei], por exemplo, podem variar de 18 a 32\% em função da suscetibilidade da cultivar e das condições climáticas (Sinclair, 1999). Para a ferrugem (Phakopsora pachyrhizi H. Sydow \& Sydow), observam-se reduções de rendimento de 10 a $90 \%$ e para o oídio [Erysiphe diffusa (Cooke \& Peck) U. Braun \& S. Takamatsu], de 10 a 35\% (Hartman et al. 1999). Nenhum desses níveis de danos é, no entanto, previsível, pois a redução de produção não tem sido consistentemente correlacionada à intensidade de doença.

Modelos tradicionais de avaliação de danos (James \& Teng, 1979) são obtidos de forma empírica, fazendo variar a quantidade de doença em diferentes parcelas e correlacionando os níveis de severidade com produção. O dano pode, assim, ser obtido por regressão linear simples, onde a intensidade de doença é a variável independente e a redução de produtividade a variável dependente. A equação que correlaciona doença e dano é denominada função de dano (Zadoks, 1985). Essa equação é necessária em programas de manejo integrado de doenças de plantas porque estima a redução na produção, para cada nível de severidade, ou seja, fornece subsídios biológicos necessários para o cálculo do limiar de dano econômico. Para alguns patossistemas, a obtenção da função de dano não é possível, uma vez que a produção vegetal é função da duração da área foliar sadia e não da área destruída pelo patógeno (Waggoner \& Berger, 1987; Bergamin Filho et al. 1995). Em culturas onde o número de folhas é fixo, como nos cereais, área foliar sadia e área foliar doente são altamente correlacionadas e o aumento da quantidade de área doente necessariamente implica em redução na área sadia. Por essa razão, a função de dano tem sido usada com êxito em cereais (Reis et al., 2000). Funções de danos para doenças foliares em leguminosas são escassas, parcialmente em função dos complexos mecanismos de formação da produção nessas culturas (Gaunt, 1987).

Este trabalho foi desenvolvido com o objetivo de utilizar a metodologia tradicional de avaliação de danos, visando quantificar as relações entre intensidade de doença, duração da área foliar sadia e produtividade, para os patossistemas soja-oídio e soja-crestamento foliar.

\section{MATERIALEMÉTODOS}

Os ensaios foram conduzidos, na safra 2002/03, em Ponta Grossa, Paraná. As cultivares semiprecoces BRS 133 e BR 16, com diferença de suscetibilidade a oídio, foram semeadas em 20 de novembro de 2002, no sistema de plantio direto, com espaçamento entre ruas de $0,45 \mathrm{~cm}$ e densidade de 12 plantas por metro linear. A adubação constou de $300 \mathrm{~kg} / \mathrm{ha}$ de adubo da fórmula 00-20-20 e a emergência das plantas ocorreu no dia 28 de novembro. $\mathrm{O}$ delineamento experimental utilizado foi blocos ao acaso com seis tratamentos (diferentes níveis de doença) e quatro repetições, sendo cada parcela constituída por cinco linhas de $8 \mathrm{~m}$.

Para a obtenção dos diferentes níveis de doença, foram utilizadas aplicações da mistura pronta do fungicida com os ingredientes ativos (i.a.) difenoconazole 250 g i.a./litro + propiconazole $250 \mathrm{~g}$ i.a./litro. Os tratamentos e as doses de ingrediente ativo (i.a.) utilizados foram: 1- sem aplicação; 2aplicação no estádio R3 (difenoconazole 37,5 g i.a./ha + propiconazole 37,5 g i.a./ha); 3- aplicações nos estádios R3 e R5.1 (difenoconazole 37,5 g i.a./ha + propiconazole 37,5 g i.a./ ha); 4- Aplicações nos estádios R3 e R5. 1 (difenoconazole 18,75 g i.a./ha + propiconazole 18,75 g i.a./ha); 5- aplicação no estádio R5.1 (difenoconazole 37,5 g i.a./ha + propiconazole 37,5 g i.a./ha); e 6- três aplicações com intervalos de 14 dias a partir do estádio R3 (difenoconazole 37,5 g i.a./ha + propiconazole 37,5 g i.a./ha). Os estádios R3 e R5.1 correspondem ao início de formação das vagens e início de formação das sementes, respectivamente (Fehr \& Caviness, 1981). As aplicações do fungicida foram realizadas utilizando pulverizador pressurizado com $\mathrm{CO}_{2}$, equipado com barra de 2,0 $\mathrm{m}$, contendo quatro bicos do tipo cônico XR110:02. A pressão de trabalho foi de 30 libras $/ \mathrm{pol}^{2}$, com volume de calda de $200 \mathrm{l} /$ ha.

As avaliações foram realizadas a partir do início das pulverizações, em intervalos médios semanais, quantificandose a severidade das doenças e o índice de área foliar. Para as avaliações de severidade, foi utilizada escala diagramática (Martins, 2003), quantificando as doenças presentes nos terços inferior, médio e superior das plantas, em quatro pontos na parcela, utilizando a média das severidades obtidas como a severidade da parcela. A avaliação do índice de área foliar foi realizada utilizando o analisador de dossel (LAI 2000 - Li-Cor ${ }^{\circledR}$ ), que quantifica a luz que atravessa o dossel, dado que se correlaciona com a área foliar da parcela (Chason et al., 1991). A produtividade e o peso de 100 sementes foram quantificados ao final do ciclo da cultura, nos $4 \mathrm{~m}^{2}$ centrais de cada parcela.

Foram calculadas as variáveis área abaixo da curva de progresso da doença (AACPD) e duração da área foliar sadia (DAFS). A variável AACPD foi calculada integrando a curva de progresso da doença para cada parcela, através da fórmula:

$$
A A C P D=\sum_{i}^{n-1}\left(\frac{x_{i}+x_{i+1}}{2}\right)\left(t_{i+1}-t_{i}\right)
$$

onde, $n$ é o número de avaliações, $x$ é a proporção de doença e é $\left(t_{i+1}-t_{i}\right)$ o intervalo de avaliações consecutivas.

Para o cálculo da variável DAFS, foi utilizado o índice de área foliar (IAF), estimado pelo avaliador de dossel Li-Cor ${ }^{\circledR}$, utilizando a equação:

$$
D A F S=\sum_{i=1}^{n_{0}-1}\left[\operatorname{IAF}\left(1-x_{i}\right)+I A F_{i+1}\left(1-x_{i+1}\right)\right] / 2\left(t_{i+1}-t_{i}\right)
$$

A análise estatística dos dados com o programa PlotIT (Scientific Programming Enterprises, Haslett, MI) foi realizada através de regressões lineares, entre as variáveis de doença (severidade e AACPD) e de produção, e não lineares, entre DAFS e produtividade. Os resultados obtidos nos diferentes tratamentos foram submetidos à análise de variância pelo teste F, e a diferença entre as médias, quando significativas, foram comparadas pelo teste de Tukey ao nível de 5\% de probabilidade, utilizando o programa SASM-Agri (Canteri et al., 2001). Os valores de severidade foram transformados em $\operatorname{arcsen} \sqrt{x / 100}$ para análise estatística. 


\section{C.V. Godoy \& M.G. Canteri}

\section{RESULTADOSE DISCUSSÃO}

As doenças que predominaram no ensaio, nas duas cultivares avaliadas, foram o oídio e o crestamento foliar de cercospora (C. kikuchii T. Matsu \& Tomoyasu), sendo os primeiros sintomas de oídio observados nas parcelas não tratadas aos 104 dias após o plantio, quando a cultura se encontrava no estádio de formação de grãos (R5) (Tabelas 1 e 2). O crestamento foliar de cercospora foi observado a partir dos 120 dias após o plantio, no estádio R6 (vagens com sementes verdes que preenchem totalmente a cavidade) (Fehr \& Caviness, 1981). As avaliações aos 111 e 128 dias após o plantio correspondem aos estádios R5.5 (maioria das vagens entre 75 e $100 \%$ de granação) e R7 (início a 50\% de amarelecimento de folhas e vagens), respectivamente. Todos tratamentos foram eficientes na redução da severidade das doenças, quando comparados com a média das parcelas não tratadas (Tabelas 1 e 2). Para a variedade BRS 133, o tratamento menos eficiente na redução da severidade foi à aplicação única de fungicida realizada no estádio R3 (Tabela 1), sendo estatisticamente diferente dos demais tratamentos pelo teste de Tukey ao nível de 5\% de probabilidade, aos 120 e 128 dias após o plantio. Na variedade BR 16, a menor eficiência na redução da severidade do tratamento no estádio R3 foi observada aos 120 dias após o plantio (Tabela 2) e, embora esse tratamento tenha apresentado um nível maior de severidade aos 128 dias após o plantio, quando comparado com os demais tratamentos, não mostrou diferença estatística significativa. A menor eficiência da aplicação do fungicida no estádio R3 ocorreu provavelmente porque o produto apresentava baixo residual para o controle das doenças que ocorreram de forma tardia no ciclo da cultura (Tabelas 1 e 2). Nenhum tratamento promoveu sintomas de fitotoxicidade nas cultivares testadas.

Para as duas cultivares constatou-se que a utilização de fungicidas constitui-se em uma importante ferramenta no controle de oídio e crestamento de cercospora, uma vez que a aplicação do fungicida difenoconazole + propiconazole proporcionou aumento de produtividade e no peso de 100 sementes (Tabelas 1 e 2). Para a cultivar BRS 133 os aumentos

TABELA 1 - Médias de severidade de oídio (104 e 111 dias após o plantio) e oídio + crestamento foliar de cercospora (120 e 128 dias após o plantio), área abaixo da curva de progresso de doença (AACPD), duração da área foliar sadia (DAFS), produtividade (sacas/ ha) e peso de 100 sementes (g) para os diferentes tratamentos de fungicida na cultivar de soja (Glycine max) BRS 133. Ponta Grossa, PR, safra 2002/03

\begin{tabular}{|c|c|c|c|c|c|c|c|c|}
\hline \multirow{3}{*}{ Tratamento } & \multicolumn{4}{|c|}{ Dias após o plantio } & \multirow{3}{*}{ AACPD } & \multirow{3}{*}{ DAFS } & \multirow{3}{*}{$\begin{array}{c}\text { Prod. } \\
\text { (sacas/ha) }\end{array}$} & \multirow{3}{*}{$\begin{array}{c}\text { Peso } \\
100 \text { sem. } \\
\text { (g) }\end{array}$} \\
\hline & 104 & 111 & 120 & 128 & & & & \\
\hline & \multicolumn{4}{|c|}{ Severidade (\%) } & & & & \\
\hline 1. não tratada & $19,7 \mathrm{a}$ & $31 \mathrm{a}$ & $26,7 \mathrm{a}$ & $57,5 \mathrm{a}$ & $1434 \mathrm{a}$ & $121,6 \mathrm{~b}$ & $46 \mathrm{~b}$ & $15,3 \mathrm{~b}$ \\
\hline 2. aplicação em R3 ${ }^{1}$ & $0 \mathrm{~b}$ & $0 \mathrm{~b}$ & $9,0 \mathrm{~b}$ & $21,7 \mathrm{~b}$ & $163 \mathrm{~b}$ & $142,7 \mathrm{ab}$ & $50 \mathrm{ab}$ & $15,3 \mathrm{~b}$ \\
\hline 3. aplicação em R3 e R5.1 ${ }^{1}$ & $0 \mathrm{~b}$ & $0 \mathrm{~b}$ & $3,0 \mathrm{c}$ & $13,1 \mathrm{c}$ & $77 \mathrm{~b}$ & $148,1 \mathrm{a}$ & $52 \mathrm{ab}$ & $15,7 \mathrm{ab}$ \\
\hline 4. aplicação em R3 e R5.1 ${ }^{2}$ & $0 \mathrm{~b}$ & $0 \mathrm{~b}$ & $2,7 \mathrm{c}$ & $11,5 \mathrm{~cd}$ & $69 \mathrm{~b}$ & $162,6 \mathrm{a}$ & $56 \mathrm{a}$ & $17,0 \mathrm{a}$ \\
\hline 5. aplicação em R5. $1^{1}$ & $0 \mathrm{~b}$ & $0 \mathrm{~b}$ & $1,5 \mathrm{c}$ & $12,2 \mathrm{~cd}$ & $60 \mathrm{~b}$ & $157,4 \mathrm{a}$ & $52 \mathrm{ab}$ & $16,4 \mathrm{ab}$ \\
\hline 6. três aplicações a partir de R $3^{1}$ & $0 \mathrm{~b}$ & $0 \mathrm{~b}$ & $1,2 \mathrm{c}$ & $6,2 \mathrm{~d}$ & $33 \mathrm{~b}$ & $151,3 \mathrm{a}$ & $53 \mathrm{a}$ & $16,3 \mathrm{ab}$ \\
\hline CV (\%) & 17,1 & 15,7 & 14,9 & 15,3 & 17,5 & 6,5 & 4,2 & 4,4 \\
\hline
\end{tabular}

Médias seguidas de mesma letra na coluna não diferem entre si pelo teste de Tukey a 5\% de probabilidade

${ }^{1}$ difenoconazole 37,5 g i.a./ha + propiconazole 37,5 g i.a./ha

2 difenoconazole 18,75 g i.a./ha + propiconazole 18,75 g i.a./ha

TABELA 2 - Médias de severidade de oídio (104 e 111 dias após o plantio) e oídio + crestamento foliar de cercospora (120 e 128 dias após o plantio), área abaixo da curva de progresso de doença (AACPD), produtividade (sacas/ha) e peso de 100 sementes (g) para os diferentes tratamentos de fungicida na cultivar de soja (Glycine max) BR 16. Ponta Grossa, PR, safra 2002/03

\begin{tabular}{|c|c|c|c|c|c|c|c|c|}
\hline \multirow{3}{*}{ Tratamento } & \multicolumn{4}{|c|}{ Dias após o plantio } & \multirow{3}{*}{ AACPD } & \multirow{3}{*}{ DAFS } & \multirow{3}{*}{$\begin{array}{c}\text { Prod. } \\
\text { (sacas/ha) }\end{array}$} & \multirow{3}{*}{$\begin{array}{c}\text { Peso } \\
100 \text { sem. } \\
\text { (g) }\end{array}$} \\
\hline & 104 & 111 & 120 & 128 & & & & \\
\hline & \multicolumn{4}{|c|}{ Severidade (\%) } & & & & \\
\hline 1. não tratada & $20,3 \mathrm{a}$ & $24,7 \mathrm{a}$ & $47,5 \mathrm{a}$ & $73,7 \mathrm{a}$ & $1569 \mathrm{a}$ & $99,5 \mathrm{~b}$ & $50 \mathrm{a}$ & $16,9 \mathrm{a}$ \\
\hline 2. aplicação em R3 ${ }^{1}$ & $0 \mathrm{~b}$ & $0 \mathrm{~b}$ & $17,2 \mathrm{~b}$ & $25,1 \mathrm{~b}$ & $244 \mathrm{~b}$ & $128,7 \mathrm{a}$ & $55 \mathrm{a}$ & $17,3 \mathrm{a}$ \\
\hline 3. aplicação em R3 e R5.1 ${ }^{1}$ & $0 \mathrm{~b}$ & $0 \mathrm{~b}$ & $6,1 \mathrm{c}$ & $15,7 \mathrm{~b}$ & $112 \mathrm{c}$ & $139,4 \mathrm{a}$ & $61 \mathrm{a}$ & $18,9 \mathrm{a}$ \\
\hline 4. aplicação em R3 e R5.1 ${ }^{2}$ & $0 \mathrm{~b}$ & $0 \mathrm{~b}$ & $6,2 \mathrm{c}$ & $21,7 \mathrm{~b}$ & $140 \mathrm{bc}$ & $144,5 \mathrm{a}$ & $61 \mathrm{a}$ & $19,1 \mathrm{a}$ \\
\hline 5. aplicação em R5.1 ${ }^{1}$ & $0 \mathrm{~b}$ & $0 \mathrm{~b}$ & $6,7 \mathrm{c}$ & $19,5 \mathrm{~b}$ & $135 \mathrm{c}$ & $131,5 \mathrm{a}$ & $56 \mathrm{a}$ & $18,8 \mathrm{a}$ \\
\hline 6. três aplicações a partir de $\mathrm{R} 3^{1}$ & $0 \mathrm{~b}$ & $0 \mathrm{~b}$ & $4,2 \mathrm{c}$ & $19,2 \mathrm{~b}$ & $112 \mathrm{c}$ & $141,0 \mathrm{a}$ & $57 \mathrm{a}$ & $19,2 \mathrm{a}$ \\
\hline $\mathrm{CV}(\%)$ & 17,1 & 23,2 & 23,1 & 11,1 & 12,3 & 6,0 & 9,1 & 12,3 \\
\hline
\end{tabular}

Médias seguidas de mesma letra na coluna não diferem entre si pelo teste de Tukey a 5\% de probabilidade

${ }^{1}$ difenoconazole $37,5 \mathrm{~g}$ i.a./ha + propiconazole $37,5 \mathrm{~g}$ i.a./ha

${ }^{2}$ difenoconazole 18,75 g i.a./ha + propiconazole 18,75 g i.a./ha 
Efeito da severidade de oídio e crestamento foliar de cercospora ...

de produtividade variaram de quatro a dez sacas/ha para os diferentes tratamentos e de 0,4 a 1,7 g para peso de 100 sementes. Aumentos de produtividade e peso de 100 sementes também foram observados por Lopes et al. (1998) quando utilizaram fungicidas para controle de doenças de final de ciclo, variando de $-0,8 \%$ a $12,7 \%$ para o peso de 100 sementes e de 0,3 a 22,3\% para produtividade. Hoffmann (2002) constatou efeito significativo da aplicação de fungicidas para o controle dessas doenças sobre a produtividade da soja, sendo o incremento dependente do cultivar utilizado, ou seja, aumentos de 10,7\% foram registrados na cultivar de ciclo tardio, 3,6\% na de ciclo precoce e 5,9\% na de ciclo médio. Para a cultivar BR 16, não foi observada diferença estatística significativa em relação à produtividade e ao peso de 100 sementes nos diferentes tratamentos, ao nível de 5\% de significância pelo teste de Tukey.

Os diferentes tratamentos foram eficientes para proporcionar um gradiente de severidade aos 120 e 128 dias após o plantio (Tabela 1 e 2). Essa variável apresentou relação negativa significativa ao nível de 5\% de probabilidade para as avaliações aos 120 e 128 dias após o plantio, nas duas cultivares utilizadas (Tabela 3). Da mesma forma, foram obtidas correlações negativas significativas ao nível de 5\% para a variável integral AACPD (Figura 1a-c) com coeficientes de determinação de 0,67 (probabilidade=0,046) e 0,69 (probabilidade=0,041), para as cultivares BRS 133 e BR 16, respectivamente. Embora tenha sido observada neste trabalho relação negativa entre severidade e produtividade, esse fato não foi observado por Guerzoni (2001) estudando o efeito das doenças de final de ciclo na cultura da soja, concluindo que a severidade não é um bom indicador para prever ou estimar reduções de produtividade na cultura da soja, para doenças de final de ciclo. Essa diferença nos resultados pode ter ocorrido em função da diferença de severidade observada nos trabalhos e também da época de ocorrência da doença. Para a situação avaliada neste ensaio, os resultados obtidos mostram que a abordagem tradicional de avaliação de danos, utilizando modelos de ponto crítico (severidade num determinado estádio de desenvolvimento) ou modelos integrais que relacionam danos com variáveis que representem a totalidade de uma epidemia, como a AACPD, são passíveis de serem utilizados para a cultura da soja. Yang et al. (1991) estudando o efeito da ferrugem na cultura da soja, também encontraram correlação negativa entre produtividade e AACPD. Apesar dos modelos de danos se constituírem em importantes ferramentas para

TABELA 3 - Coeficientes angulares da reta de regressão entre produtividade e severidade de oídio e crestamento foliar foliar $(y=a+b x$, onde $y$ é a produtividade em sacas/ha e $x$ a severidade da doença) e probabilidade (P), aos 120 e 128 dias após o plantio, para as cultivares de soja (Glycine max) BRS 133 e BR 16

\begin{tabular}{lccccccccc}
\hline \hline \multirow{2}{*}{$\begin{array}{l}\text { Dias após } \\
\text { o plantio }\end{array}$} & \multicolumn{4}{c}{ BRS 133 } & & \multicolumn{4}{c}{ BR 16 } \\
\cline { 2 - 6 } \cline { 6 - 8 } & $\boldsymbol{a}$ & $\boldsymbol{b}$ & $\mathbf{R}^{2}$ & $\mathbf{P}$ & & $\boldsymbol{a}$ & $\boldsymbol{b}$ & $\mathbf{R}^{2}$ & $\mathbf{P}$ \\
\hline 120 & 53,5 & $-0,28$ & 0,71 & $0,018^{*}$ & & 59,3 & $-0,19$ & 0,69 & $0,039^{*}$ \\
128 & 54,5 & $-0,14$ & 0,72 & $0,030^{*}$ & & 60,7 & $-0,14$ & 0,65 & $0,049^{*}$ \\
\hline *significativo a 5\% de probabilidade & & & &
\end{tabular}

entender o mecanismo de redução de produtividade, sua utilização é limitada em função de terem sido obtidos a partir de dados empíricos. Muitos dos modelos empíricos desenvolvidos para quantificação de danos, não passam no teste de transportabilidade (Rouse, 1988).

Embora os resultados tenham mostrado relação entre doença e produtividade, essa relação é bastante questionável, principalmente porque a máxima produção possível é diferente para cada campo, região ou estação de cultivo, devido a fatores edafo-climáticos. Estudos de fisiologia vegetal demonstram que a produção de uma cultura está intimamente relacionada com a porção de radiação que as plantas conseguem aproveitar e essa radiação pode não estar necessariamente associada à severidade de doença. Nessa linha, os conceitos de duração de área foliar sadia foram propostos como melhores indicadores de produção de uma cultura (Bergamin Filho et al., 1995). A variável DAFS mostrou correlação positiva altamente significativa $(\mathrm{P}<0,001)$ com a produtividade (quanto maior a duração da área foliar, maior a produtividade), para as duas cultivares. O modelo monomolecular

$$
y=b_{1} *\left(1-b_{2} \exp \left(-b_{3}^{*} x\right)\right)
$$

onde $y$ é a produtividade em sacas/ha, $x$ a DAFS e $b_{1}, b_{2}$ e $b_{3}$ são parâmetros do modelo, foi ajustado aos dados, com coeficientes de determinação de 0,76 e 0,62, para as cultivares BRS 133 e BR 16, respectivamente (Figura 1b-d). A redução na duração da área foliar sadia na cultura da soja ocorre não só devido à área lesionada pelo patógeno, como também pela desfolha precoce provocada pelas doenças. A redução do valor de DAFS para a parcela sem tratamento reflete o menor índice de área foliar nas avaliações associado à área lesionada pelos patógenos (Tabelas 1 e 2). Todos os tratamentos com difenoconazole + propiconazole apresentaram maior duração da área foliar sadia, quando comparados com as parcelas não tratadas, para as duas cultivares avaliadas. Guerzoni (2001) estudando o efeito da mancha parda (Septoria glycines Hemmi) e crestamento foliar de cercospora em soja constatou relações significativas e positivas da duração da área foliar sadia e produtividade. Madeira et al. (1988), analisando o patossistema Ascochyta fabae Speg. - Vicia faba L., observaram que parcelas com plantas infetadas mostraram queda mais rápida no índice de área foliar do que as plantas sadias, sugerindo a senescência precoce. O principal dano atribuído às doenças foliares que incidem no final do ciclo da cultura da soja é a desfolha precoce, que encurta o ciclo da cultura, prejudicando o enchimento das vagens e a completa formação dos grãos (Hartman et al., 1999). Variáveis de área foliar (DAFS) têm sido bastante utilizadas em patossistemas onde a severidade da doença não correlaciona com a produtividade, como é o caso das doenças foliares do feijoeiro (Phaseolus vulgaris L.) (Bergamin Filho et al., 1995).

No ensaio conduzido em Ponta Grossa com as cultivares BRS 133 e BR 16, a variável injúria nos estádios R6 e R7 (expressa em severidade de doença) e AACPD foram utilizadas com sucesso para explicar a redução de produtividade (Tabela 3 e Figura 1). A mistura pronta do fungicida com os ingredientes ativos difenoconazole 250 g i.a./l + propiconazole 250 g i.a./l 
C.V. Godoy \& M.G. Canteri
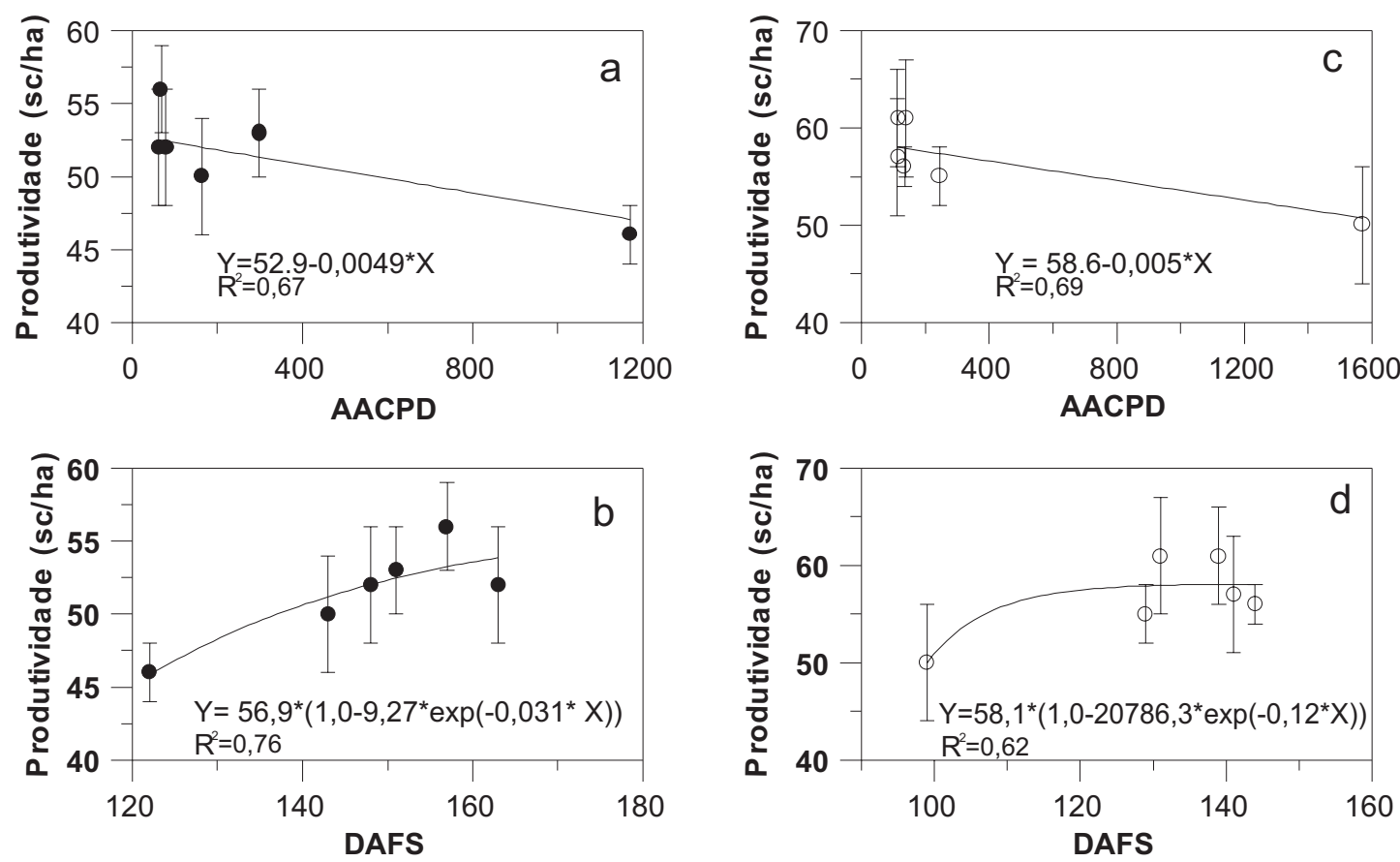

FIG 1 - Relação entre produtividade (sacas/ha), AACPD e DAFS para as cultivares de soja (Glycine max) BRS 133 (a-b) e BR 16 (c-d).

foi eficiente no controle do oídio e do crestamento foliar de cercopora, nos tratamentos e doses testadas, apresentado menor eficiência em aplicação única no estádio R3. A variável DAFS explicou a resposta em produtividade, pois engloba o efeito do patógeno no tecido foliar e a desfolha antecipada provocada pela doença. Trabalhos de avaliação de danos para outras doenças foliares devem ser realizados para a implementação de um sistema de manejo integrado de doenças que associe injúria, variáveis de doença e de área foliar sadia, na cultura da soja.

\section{REFERÊNCIAS BIBLIOGRÁFICAS}

BERGAMIN FILHO, A., LOPES, D.B., AMORIM, L., GODOY, C.V. \& BERGER, R.D. Avaliação de danos causados por doenças de plantas. Revisão Anual de Patologia de Plantas 3:132-185. 1995.

CANTERI, M.G., ALTHAUS, R.A., VIRGENS FILHO, J.S., GIGLIOTI, E.A. \& GODOY, C.V. SASM-Agri: Sistema para análise e separação de médias em experimentos agrícolas pelos métodos ScottKnott, Tukey e Duncan. Revista Brasileira de Agrocomputação 1:1824. 2001.

CANTERI, M.G. \& GODOY, C.V. Escala diagramática para avaliação da severidade da ferrugem da soja. Summa Phytopathologica 29:89. 2003 (Resumo).

CHASON, J.W., BALDOCCHI, D.D. \& HUSTON, M.A. A comparison of direct and indirect methods for estimating forest canopy leaf area. Agricultural and Forest Meteorology 57:107-128. 1991.

CONAB. Previsão e acompanhamento da safra 2002/03. Quinto levantamento. www.conab.gov.br. 2003

EMPRESA BRASILEIRA DE PESQUISA AGROPECUÁRIA. Centro Nacional de Pesquisa de Soja. Tecnologia de produção da soja
- Região Central do Brasil, 2003. Londrina: Embrapa Soja; Embrapa Cerrados; Embrapa Agropecuária Oeste; ESALQ, Embrapa Soja/ Sistemas de Produção 1, 2002.

FEHR, W.R. \& CAVINESS, C.E. Stage of soybean development. Iowa State University. Special report 80, March, 1981.

GAUNT, R.E. A mechanistic approach to yield loss assessment based on crop physiology. In: Teng, P.S. (Ed.) Crop loss assessment and pest management St Paul. APS Press. 1987. pp.150-159.

GUERZONI, R.A. Efeito das doenças foliares de final de ciclo (Septoria glycines Hemmi e Cercospora kikuchii (Matsu \& Tomoyasu) Gardner) na duração da área foliar sadia da soja. (Tese de Mestrado). Piracicaba. Universidade de São Paulo. 2001

HARTMAN, G.L., SINCLAIR, J.B. \& RUPE, J.C. Compendium of soybean diseases. 4. ed. St. Paul, Minnesota:APS Press. 1999.

HOFFMANN, L.L. Controle de oídio e doenças de final de ciclo em soja. (Tese de Mestrado). Passo Fundo. Universidade de Passo Fundo. 2002

JAMES, W.C. \& TENG, P.S. The quantification of production constraints associated with plant diseases. Applied Biology 4:201267. 1979.

JAMES, W.C., JENKINS, J.E.E. \& JEMMETT, J.L. The relationship between leaf blotch caused by Rhynchosporium secalis and losses in grains yield of spring barley. Annals of Applied Biology 62:273-288. 1968.

LOPES, M.E.B.M., KLEIN-GUNNEWIECK, R.A., BARROS, B.C. \& SINIGACLIA, C. Controle químico da mancha parda e crestamento foliar da soja (Glycine max (L.) Merril). Revista de Agricultura 73:2330. 1998.

MADEIRA, A.C., CLARK, J.A. \& ROSSALL, S. Growth, light interception and disease in field bean (Vicia faba): the effect of late infection by Ascochyta fabae. Annals of Applied Biology 112:585- 
95. 1988.

MARTINS, M.C. Produtividade da soja sob influência de ocorrência natural de Septoria glycines Hemmi e Cercospora kikuchii (Matsuo. \& Tomoyasu) Gardner com e sem controle químico. (Tese de Doutorado). Piracicaba. Escola Superior de Agricultura Luiz de Queiroz. 2003.

REIS, E.M., CASA, R.T., HOFFMANN, L.L. \& MENDES, C.M. Effect of leaf rust on wheat grain yield. Fitopatologia Brasileira 25:6771. 2000.

ROUSE, D.I. Use of crop growth-models to predict the effects of disease. Annual Review of Phytopathology 26:183-201. 1988.
SINCLAIR, J.B. Target spot. In:Hartman, G.L., Sinclair, J.B. \& Rupe, J.C. Compendium of soybean diseases. 4. ed. St. Paul: APS Press, 1999.

WAGGONER, P.E. \& BERGER, R.D. Defoliation, disease and growth. Phytopathology 77:393-398. 1987.

YANG, X.B., TSCHANZ, A.T., DOWLER, W.M. \& WANG, T.C. Development of yield loss models in relation to reductions of components of soybean infected with Phakopsora pachyrhizi. Phytopathology 81:1420-1426. 1991.

ZADOKS, J.C. On the conceptual basis of crop loss assessment: the threshold theory: Annual Review of Phytopathology 23:455-473. 1985. 